\title{
Synthesis and Crystal Structure of the First 6a-Thiathiophthen Metal Complex $\left[\mathrm{Mo}(\mathrm{CO})_{5} \mathrm{PPh}_{2}\right]_{2}\left(\mu-\mathrm{C}_{5} \mathrm{H}_{2} \mathrm{~S}_{3}\right)$
}

\author{
Kuang-Hway Yih, Ying-Chih Lin,* Gene-Hsiang Lee and Yu Wang \\ Department of Chemistry, National Taiwan University, Taiwan 106, Republic of China
}

The first 6a-thiathiophthen metal complex was prepared by treating $\mathrm{M}(\mathrm{CO})_{5}\left[\mathrm{PPh}_{2} \mathrm{CS}_{2} \mathrm{CH}_{2} \mathrm{C} \equiv \mathrm{CH}\right]$ with a catalytic amount of secondary amine or tertiary amine; the structure of the 6a-thiathiophthen molybdenum complex is confirmed by an $\mathrm{X}$-ray diffraction analysis.

With their unusually long S-S distances and possible aromatic properties of the two fused five-membered rings, the 6athiathiophthen (3,3a,4-trithiopentalene) derivatives ${ }^{1}$ have attracted considerable attention. Methods for the synthesis of $6 \mathrm{a}-$ thiathiophthen ${ }^{2}$ and arylthio- or alkylthio-derivatives ${ }^{3}$ using various thionation reagents such as $\mathrm{S}, \mathrm{H}_{2} \mathrm{~S}$ and $\mathrm{P}_{2} \mathrm{~S}_{5}$ have been reported. Furthermore, much work has been done on the bonding, ${ }^{4}$ structure, ${ }^{5}$ reactions ${ }^{6}$ and electron density deformation $^{7}$ studies on 6a-thiathiophthen and its derivatives. However, no 6a-thiathiophthen metal complex has been reported. Here we report the high yield synthesis and the structure determination of the first 6a-thiathiophthen metal complex, which was prepared from the metal complex containing the diphenyl $(S$ prop-2-ynyl-dithioformato)phosphine ligand. ${ }^{8}$

Treatment of $\mathrm{M}(\mathrm{CO})_{5}\left[\mathrm{PPh}_{2}\left(\mathrm{CS}_{2} \mathrm{CH}_{2} \mathrm{C} \equiv \mathrm{CH}\right)\right](\mathrm{M}=\mathrm{Mo}, \mathbf{1 a}$; W, 1 b) with a catalytic amount of $\mathrm{Et}_{3} \mathrm{~N}$ in $\mathrm{CH}_{2} \mathrm{Cl}_{2}$ yields the $6 \mathrm{a}-$ thiathiophthen metal complexes $\left[\mathrm{M}(\mathrm{CO})_{5} \mathrm{PPh}_{2}\right]_{2}\left(\mu-\mathrm{C}_{5} \mathrm{H}_{2} \mathrm{~S}_{3}\right)(\mathrm{M}$ $=\mathrm{Mo}, \mathbf{2} \mathbf{a} ; \mathrm{W}, \mathbf{2 b})$ at room temperature (Scheme 1). Complex $\mathbf{2 a}$ is isolated as a red microcrystalline powder by recrystallization from hexane- $\mathrm{CH}_{2} \mathrm{Cl}_{2}$ in $\mathrm{ca}$. $85 \%$ yield. The spectroscopic $\dagger$ and analytical data of $\mathbf{2 a}$ are in agreement with the formulation. The FAB mass spectrum of 2 a shows a base peak at $\mathrm{m} / \mathrm{z} 721$, corresponding to $\left[\mathrm{MoPPh}_{2}\right]_{2}\left(\mu-\mathrm{C}_{5} \mathrm{H}_{2} \mathrm{~S}_{3}\right)^{+}$, formed by loss of the ten $\mathrm{CO}$ groups from $2 \mathbf{a}$. The IR spectrum of $\mathbf{2 a}$ shows two terminal carbonyl stretches at 2073 and $1924 \mathrm{~cm}^{-1}$, a typical pattern for a $\mathrm{LM}(\mathrm{CO})_{5}$ unit in octahedral geometry. The ${ }^{1} \mathrm{H}$ NMR spectrum of $2 \mathbf{a}$ exhibits a doublet at $\delta 7.90\left({ }^{3} J_{\mathrm{P}-\mathrm{H}}=7.2\right.$ $\mathrm{Hz}$ ) attributed to the two equivalent methyne protons, and the corresponding ${ }^{13} \mathrm{C}$ NMR signal is a doublet at $\delta 177.95\left({ }^{2} J_{\mathrm{P}-\mathrm{C}}\right.$ $=11.3 \mathrm{~Hz}$ ). The low field ${ }^{1} \mathrm{H}$ chemical shift is regarded as evidence for a strong ring current. The ${ }^{13} \mathrm{C}$ NMR resonance of the 3a-carbon exhibits a triplet at $\delta 177.21\left({ }^{3} J_{\mathrm{P}-\mathrm{C}}=8.3 \mathrm{~Hz}\right)$. The ${ }^{1} \mathrm{H}$ and ${ }^{13} \mathrm{C}$ NMR spectra clearly imply $C_{2 v}$ symmetry in $\mathbf{2 a}$. The molecular structure of this unusual complex $\mathbf{2 a}$ is confirmed by an X-ray diffraction study $¥$ An ORTEP drawing of $2 \mathbf{a}$ is shown in Fig. 1. The coordination geometry about the two molybdenum atoms can be described as distorted octahedral. Two metal atoms were bridged by two phosphorus atoms connected by a 6 a-thiathiophthen unit. The two $\mathrm{S}-\mathrm{S}$ distances [2.318(2) and 2.330(2) $\AA]$ in complex $\mathbf{2 a}$ are significantly longer than the expected S-S single bond lengths $(2.05 \AA)$ but considerably shorter than sum of the sulfur van der Waals radii $(3.7 \AA) .{ }^{9}$ Interestingly, in the crystal, $2 \mathrm{a}$ does not exhibit $C_{2 v}$ symmetry and the $\mathrm{P}_{2} \mathrm{C}_{5} \mathrm{H}_{2} \mathrm{~S}_{3}$ unit is not planar. To our

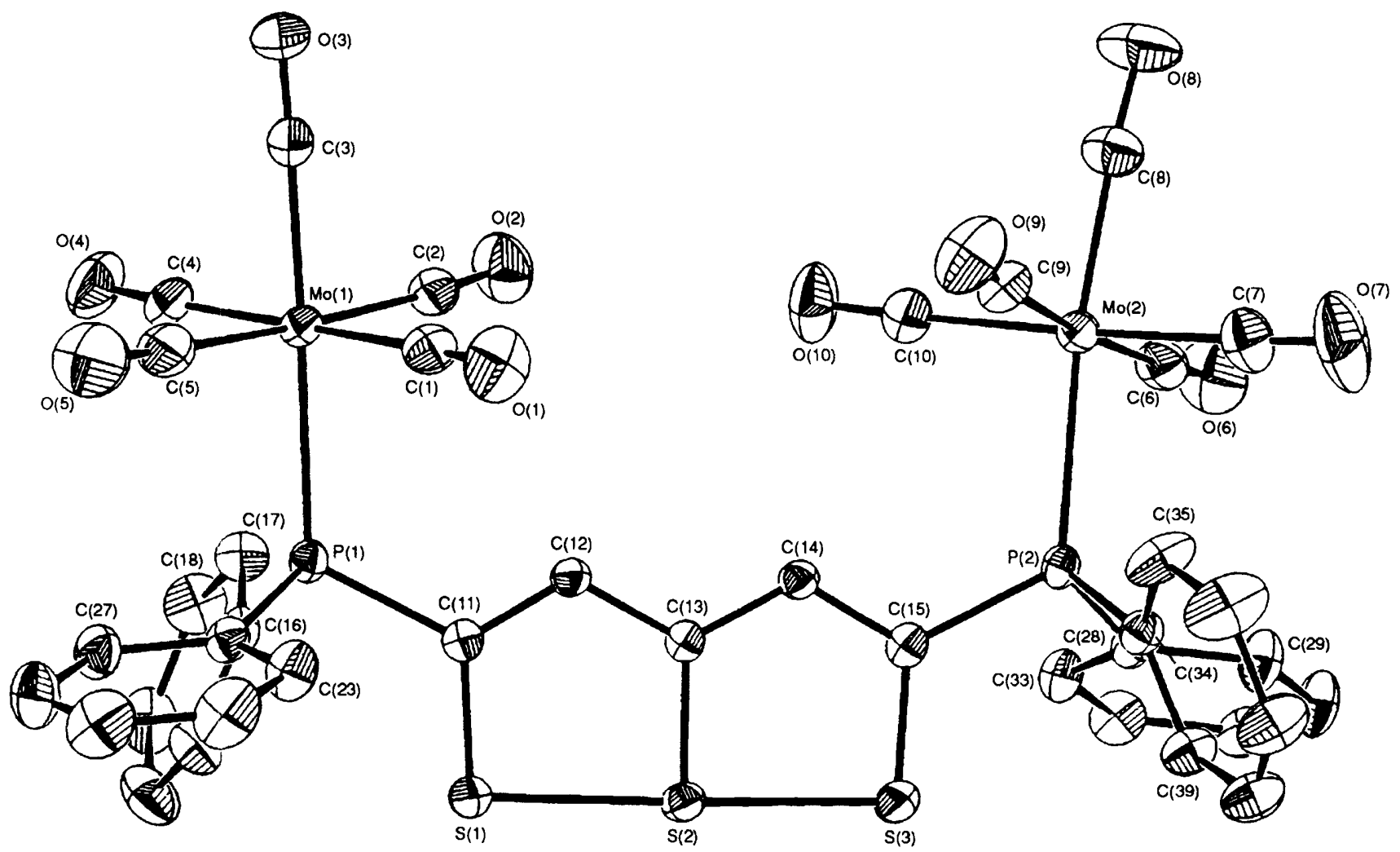

Fig. 1 ORTEP drawing for the complex $\left[\mathrm{Mo}(\mathrm{CO})_{5} \mathrm{PPh}_{2}\right]_{2}\left(\mu-\mathrm{C}_{5} \mathrm{H}_{2} \mathrm{~S}_{3}\right)$, 2a. Selected bond distances $(\AA)$ and angles $\left(^{\circ}\right)$ are as follows: Mo(1)-P(1) 2.528(2), Mo(2)-P(2) 2.540(2), P(1)-C(11) 1.855(4), P(2)-C(15) 1.847(4), C(11)-S(1) 1.689(4), C(13)-S(2) 1.736(4), C(15)-S(3) 1.689(4), C(11)$\mathrm{C}(12) 1.352(5), \mathrm{C}(12)-\mathrm{C}(13)$ 1.415(5), C(13)-C(14) 1.399(5), C(14)-C(15) 1.371(5), S(1)-S(2) 2.318(2), S(2)-S(3) 2.330(2); C(11)-P(1)-Mo(1) 118.88(13), C(15)-P(2,-Mo(2) 121.54(13), S(1)-S(2)-S(3) 178.32(6). 


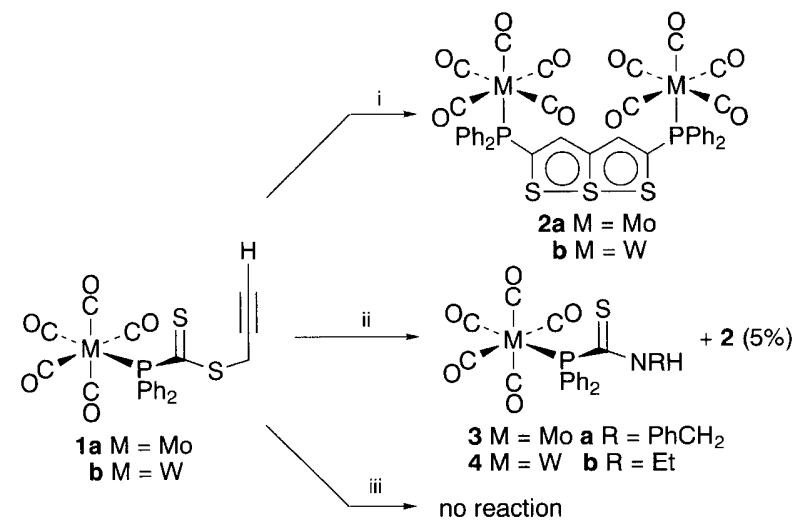

Scheme 1 Reagents and conditions: $\mathrm{i}, \mathrm{Et}_{3} \mathrm{~N}$ or $\mathrm{Pr}_{2}{ }_{2} \mathrm{NH}$ or $\mathrm{Et}_{2} \mathrm{NH}$ or $\mathrm{F}^{-}$, $\mathrm{CH}_{2} \mathrm{Cl}_{2}, 25^{\circ} \mathrm{C}, 10 \mathrm{~min}$; ii, $\mathrm{RNH}_{2}\left(\mathrm{R}=\mathrm{PhCH}_{2}, \mathrm{Et}\right), \mathrm{CH}_{2} \mathrm{Cl}_{2}, 25^{\circ} \mathrm{C}$, $1 \mathrm{~min}$; iii, $\mathrm{Bu}{ }^{\mathrm{nLi}}$ or $\mathrm{Bu}^{\mathrm{t}} \mathrm{OK}$ or $\mathrm{PhNH}_{2}, \mathrm{THF}, 25^{\circ} \mathrm{C}, 1 \mathrm{~h}$

knowledge, complex $\mathbf{2 a}$ is the first example of metal-derivative of 6a-thiathiophthen.

In order to study the role of $\mathrm{Et}_{3} \mathrm{~N}$ in the formation of $\mathbf{2 a}$, other amines and $\mathrm{Bu}_{4} \mathrm{NF}$ were used to replace $\mathrm{Et}_{3} \mathrm{~N}$ in the reaction. Complexes 1a and $1 \mathbf{b}$ were reacted with secondary amine $\left(\operatorname{Pr}_{2}{ }_{2} \mathrm{NH}, \mathrm{Et}_{2} \mathrm{NH}\right)$ or $\mathrm{Bu}^{\mathrm{n}}{ }_{4} \mathrm{NF}$ to give $\mathbf{2 a}$ and $\mathbf{2 b}$, respectively, both in high yield. The rate of formation of 2 depends on the amine used and decreases in the order $\mathrm{Et}_{3} \mathrm{~N}>\mathrm{Pr}_{2}{ }_{2} \mathrm{NH}>\mathrm{Et}_{2} \mathrm{NH}$ $>\mathrm{Bu}_{4}{ }_{4} \mathrm{NF}$. No reaction was observed when 1 was reacted with $\mathrm{Bu}{ }^{\mathrm{nLi}}$, ButOK or $\mathrm{PhNH}_{2}$. But the reactions of 1 with several primary aliphatic amines $\left(\mathrm{RNH}_{2} ; \mathrm{R}=\mathrm{PhCH}_{2}\right.$, Et $)$ give $\mathrm{M}(\mathrm{CO})_{5} \mathrm{PPh}_{2} \mathrm{CSNHR}\left(\mathrm{M}=\mathrm{Mo}, \mathrm{R}=\mathrm{PhCH}_{2}, \mathrm{Et} ; \mathbf{3 a}-\mathbf{b} ; \mathrm{M}=\right.$ $\left.\mathrm{W}, \mathrm{R}=\mathrm{PhCH}_{2}, \mathrm{Et} ; \mathbf{4 a - b}\right)$ and $\mathrm{HC} \equiv \mathrm{CCH}_{2} \mathrm{SH}$ in high yield, Scheme 1. Interestingly, complex 3 (or 4 ) is not the precusor that leads to 2 . On the basis of the above-mentioned experiments, one can conclude that secondary or tertiary amines catalyse the formation of $\mathbf{2}$ but primary amines or strong bases do not. To probe the origin of the two methyn protons of $\mathbf{2 a}$ (from the terminal or the methylene of 1a), ${ }^{2} \mathrm{H}$-labelling experiment was carried out. Treatment of the terminally labelled $\left[{ }^{2} \mathrm{H}_{1}\right] 1 \mathbf{a}$ with $\mathrm{Et}_{3} \mathrm{~N}$ afforded $2 \mathrm{a}$ with $n o^{2} \mathrm{H}$-labelling. In addition, when the reaction was monitored by the ${ }^{31} \mathrm{P}$ and ${ }^{1} \mathrm{H}$ NMR spectra, complex $\mathbf{2 a}$ was observed as the only product (yield 95\% from integration of the ${ }^{31}$ P NMR spectrum) and no intermediate was observed. Attempts to trap possible intermediates by separate addition of $\mathrm{PPh}_{3}, \mathrm{CS}_{2}, \mathrm{TCNE}$, MeI or cyclopentadiene into the reaction of 1 with $\mathrm{Et}_{3} \mathrm{~N}$ failed to produce any product other than 2 . The metal carbonyl fragment is crucial for the formation of 2 , since treatment of the analogous organic species $\mathrm{Et}_{2} \mathrm{NC}(\mathrm{S}) \mathrm{SCH}_{2} \mathrm{C} \equiv \mathrm{CH}$ with $\mathrm{Et}_{3} \mathrm{~N}$ or $\mathrm{PhCH}_{2} \mathrm{NH}_{2}$ resulted in no reaction under the same reaction conditions. In the absence of $\mathrm{R}_{3} \mathrm{~N}$, dimerization of 1 gave a fivemembered ring consisting of a $\mathrm{C}=\mathrm{S}$ unit and the propynyl moiety. ${ }^{10}$

The reactivity of the $6 \mathrm{a}$-thiathiophthen metal complexes and the mechanism for their formation are currently under investigation.

We thank the National Science Council of Taiwan, the Republic of China for support.

Received, 2nd November 1994; Com. 4/06708H

\section{Footnotes}

† Selected spectroscopic data: ${ }^{1} \mathrm{H}(300 \mathrm{MHz})$ and ${ }^{13} \mathrm{C}\left\{{ }^{1} \mathbf{H}\right\}(75 \mathrm{MHz})$ NMR ( $298 \mathrm{~K}, \mathrm{CDCl}_{3}$, relative to $\mathrm{SiMe}_{4}$, multiplicity, assignment, $J$ in $\mathrm{Hz}){ }^{31} \mathrm{P}(121.5 \mathrm{MHz}) \mathrm{NMR}\left(\mathrm{H}_{3} \mathrm{PO}_{4}\right.$ external standard $)$.

1a: IR $\left(\mathrm{CH}_{2} \mathrm{Cl}_{2}, v_{\mathrm{CO}} / \mathrm{cm}^{-1}\right): 2075(\mathrm{~m}), 1942(\mathrm{vs}) .{ }^{31} \mathrm{P}$ NMR: $\delta 76.76$. ${ }^{1} \mathrm{H}$ NMR: $\delta 2.18\left(\mathrm{t}, 1 \mathrm{H}, \equiv \mathrm{CH},{ }^{4} J_{\mathrm{H}-\mathrm{H}}=2.68\right), 3.98\left(\mathrm{~d}, 2 \mathrm{H}, \mathrm{S}-\mathrm{CH}_{2},{ }^{4} J_{\mathrm{H}-\mathrm{H}}\right.$
$=2.68), 7.47(\mathrm{~m}, 6 \mathrm{H}, \mathrm{Ph}), 7.67(\mathrm{~m}, 4 \mathrm{H}, \mathrm{Ph}) .{ }^{13} \mathrm{C}$ NMR: $\delta 26.36(\mathrm{~S}-$ $\left.\mathrm{CH}_{2}\right), 72.60(\equiv C \mathrm{H}), 75.77(\mathrm{C} \equiv \mathrm{CH}), 128.55\left(\mathrm{~d}\right.$, meta $-\mathrm{C}$ of $\mathrm{Ph},{ }^{3} J_{\mathrm{P}-\mathrm{C}}=$ $9.60), 131.03(\mathrm{~s}$, para-C of $\mathrm{Ph}), 133.69\left(\mathrm{~d}\right.$, ortho $-\mathrm{C}$ of $\mathrm{Ph},{ }^{2} J_{\mathrm{P}-\mathrm{C}}=$ $11.77), 133.67$ (d, ipso-C of $\left.\mathrm{Ph}, J_{\mathrm{P}-\mathrm{C}}=30.70\right), 205.35\left(\mathrm{~d}, C \mathrm{O},{ }^{2} J_{\mathrm{P}-\mathrm{C}}=\right.$ 8.48), 209.84 (d, $\left.C S_{2}, J_{\mathrm{P}-\mathrm{C}}=26.10\right)$. MS (FAB, NBA, $\left.m / z\right): 539\left(\mathrm{M}^{+}\right)$, $483\left(\mathrm{M}^{+}-2 \mathrm{CO}\right)$

$\left[{ }^{2} \mathrm{H}_{1}\right] \mathbf{1 b}\left({ }^{2} \mathrm{H} 98 \%\right):$ IR $\left(\mathrm{CH}_{2} \mathrm{Cl}_{2}, v_{\mathrm{CO}} / \mathrm{cm}^{-1}\right): 2072(\mathrm{~m}), 1940(\mathrm{vs}),{ }^{31} \mathrm{P}$ NMR: $\delta 59.95\left(J_{\mathrm{W}-\mathrm{P}}=237.0\right) .{ }^{1} \mathrm{H}$ NMR: $\delta 3.96\left(\mathrm{~s}, 2 \mathrm{H}, \mathrm{S}-\mathrm{CH}_{2}\right), 7.45$ $(\mathrm{m}, 6 \mathrm{H}, \mathrm{Ph}), 7.69(\mathrm{~m}, 4 \mathrm{H}, \mathrm{Ph})$.

2a: IR (KBr, $\left.v_{\mathrm{CO}} / \mathrm{cm}^{-1}\right)$ : 2073(m), 1924(vs). ${ }^{31} \mathrm{P}$ NMR: $\delta 46.59 .{ }^{1} \mathrm{H}$ NMR: $\delta 7.41(\mathrm{~m}, 6 \mathrm{H}, \mathrm{Ph}), 7.52(\mathrm{~m}, 4 \mathrm{H}, \mathrm{Ph}), 7.90\left(\mathrm{~d}, 2 \mathrm{H}, \mathrm{CH},{ }^{3} J_{\mathrm{P}-\mathrm{H}}=\right.$ 7.2). ${ }^{13} \mathrm{C}$ NMR: $\delta 128-134(\mathrm{Ph}), 177.21\left(\mathrm{t}, \mathrm{HCC},{ }^{3} J_{\mathrm{P}-\mathrm{C}}=8.3\right), 177.95$ $\left(\mathrm{d}, C \mathrm{H},{ }^{2} J_{\mathrm{P}-\mathrm{C}}=11.3\right), 205.22\left(\mathrm{~d}\right.$, cis-CO,$\left.{ }^{2} J_{\mathrm{P}-\mathrm{C}}=9.0\right), 209.67(\mathrm{~d}, \mathrm{PCS}$, $\left.J_{\mathrm{P}-\mathrm{C}}=24.8\right)$. MS $(\mathrm{FAB}, \mathrm{NBA}, m / z): 1000.9\left(\mathrm{M}^{+}\right), 972.9\left(\mathrm{M}^{+}-\mathrm{CO}\right)$, $945\left(\mathrm{M}^{+}-2 \mathrm{CO}\right), 916.9\left(\mathrm{M}^{+}-3 \mathrm{CO}\right), 721.0\left(\mathrm{M}^{+}-10 \mathrm{CO}\right)$.

2b: IR (KBr, $\left.v_{\mathrm{CO}} / \mathrm{cm}^{-1}\right): 2068(\mathrm{~m}), 1931(\mathrm{vs}) .{ }^{31} \mathrm{P}$ NMR: $\delta 28.54$ $\left(J_{\mathrm{W}-\mathrm{P}}=249.6\right) .{ }^{1} \mathrm{H}$ NMR: $\delta 7.41(\mathrm{~m}, 6 \mathrm{H}, \mathrm{Ph}), 7.52(\mathrm{~m}, 4 \mathrm{H}, \mathrm{Ph}), 7.91$ $\left(\mathrm{d}, 2 \mathrm{H}, \mathrm{CH},{ }^{3} J_{\mathrm{P}-\mathrm{H}}=8.0\right) .{ }^{13} \mathrm{C}$ NMR: $\delta 128-134(\mathrm{Ph}) ; 177.21(\mathrm{t}, \mathrm{HCC}$, $\left.{ }^{3} J_{\mathrm{P}-\mathrm{C}}=8.3\right), 177.30\left(\mathrm{~d}, C \mathrm{H},{ }^{2} J_{\mathrm{P}-\mathrm{C}}=11.3\right), 196.80\left(\mathrm{~d}, c i s-C \mathrm{O},{ }^{2} J_{\mathrm{P}-\mathrm{C}}=\right.$ 9.0). MS (FAB, NBA, $m / z): 1176.2\left(\mathrm{M}^{+}\right), 1148.0\left(\mathrm{M}^{+}-\mathrm{CO}\right), 1064.0$ $\left(\mathrm{M}^{+}-4 \mathrm{CO}\right), 1036.8\left(\mathrm{M}^{+}-5 \mathrm{CO}\right), 1008.2\left(\mathrm{M}^{+}-6 \mathrm{CO}\right), 952.1\left(\mathrm{M}^{+}-\right.$

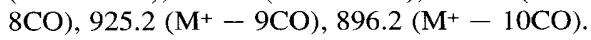

3a: ${ }^{31}$ P NMR: $\delta 63.47 .{ }^{1} \mathrm{H}$ NMR: $\delta 4.87\left(\mathrm{~s}, 2 \mathrm{H}, \mathrm{CH}_{2}\right), 7.14-7.65(\mathrm{~m}$, $15 \mathrm{H}, \mathrm{Ph})$. MS (FAB, NBA, $m / z): 571.4\left(\mathrm{M}^{+}\right), 543.4\left(\mathrm{M}^{+}-\mathrm{CO}\right)$.

3b: ${ }^{11} \mathrm{P}$ NMR: $\delta 62.38$. ${ }^{1} \mathrm{H}$ NMR: $\delta 1.14\left(\mathrm{t}, 6 \mathrm{H}, \mathrm{CH}_{3}, J_{\mathrm{H}-\mathrm{H}}=7.3\right)$, $3.68\left(\mathrm{q}, 4 \mathrm{H}, \mathrm{CH}_{2}, J_{\mathrm{H}-\mathrm{H}}=7.3\right), 2.42(\mathrm{~b}, 1 \mathrm{H}, \mathrm{N} H), 7.43-7.67(\mathrm{~m}, 10 \mathrm{H}$, $\mathrm{Ph})$.

4a: ${ }^{31} \mathrm{P}$ NMR: $\delta 47.14\left(J_{\mathrm{W}-\mathrm{P}}=256.4\right) .{ }^{1} \mathrm{H}$ NMR: $\delta 4.86(\mathrm{~s}, 2 \mathrm{H}$, $\left.\mathrm{CH}_{2}\right), 7.29-7.68(\mathrm{~m}, 15 \mathrm{H}, \mathrm{Ph}), 7.91\left(\mathrm{~d}, 2 \mathrm{H}, \mathrm{CH},{ }^{3} J_{\mathrm{P}-\mathrm{H}}=8.0\right)$. MS (FAB, NBA, $m / z): 659.3\left(\mathrm{M}^{+}\right), 631.3\left(\mathrm{M}^{+}-\mathrm{CO}\right)$.

4b: ${ }^{31} \mathrm{P}$ NMR: $\delta 46.30\left(J_{\mathrm{W}-\mathrm{P}}=257.6\right) .{ }^{1} \mathrm{H}$ NMR: $\delta 1.19\left(\mathrm{t}, 6 \mathrm{H}, \mathrm{CH}_{3}\right.$, $\left.J_{\mathrm{H}-\mathrm{H}}=7.3\right), 3.95\left(\mathrm{q}, 4 \mathrm{H}, \mathrm{CH}_{2}, J_{\mathrm{H}-\mathrm{H}}=7.3\right), 7.30-7.63(\mathrm{~m}, 10 \mathrm{H}$, $\mathrm{Ph})$.

$\ddagger$ Crystal data for $2 \mathrm{a}: \mathrm{C}_{39} \mathrm{H}_{22} \mathrm{O}_{10} \mathrm{P}_{2} \mathrm{~S}_{3} \mathrm{Mo}_{2}$, space group $P \overline{1}, a=$ 9.042(7), $b=15.175(6), c=16.554(8) \AA, \alpha=112.02(4), \beta=$ 96.38(4), $\gamma=92.92(4)^{\circ}, V=2082.2(21) \AA^{3}, Z=2, D_{\text {calcd }}=1.596$ $\mathrm{g} \mathrm{cm}^{-3}, \mu=8.603 \mathrm{~cm}^{-1}$, observed reflections $4298,2 \theta_{\max }=45.0^{\circ}$. An absorption correction has been carried out. The structure was solved by Patterson synthesis then refined via standard least-squares and difference Fourier techniques. Non-hydrogen atoms were refined by using anisotropic thermal parameters. Total number of parameters: 506 . $R=0.028, R_{\mathrm{w}}=0.029 ; \mathrm{GOF}=1.36, \Delta F=0.51,-0.48 \mathrm{e}^{3}$; Atomic coordinates, bond lengths and angles, and thermal parameters have been deposited at the Cambridge Crystallographic Data Centre. See Information for Authors, Issue No. 1.

\section{References}

1 Y. Wang, S. K. Yeh, S. Y. Wu, C. T. Pai, C. R. Lee and K. J. Lin, Acta Crystallogr., Sect. B: Struct. Sci., 1991, 47, 298; L. K. Hansen and A. Hordvik, Acta. Chem. Scand., 1973, 27, 411; Q. Shen and K. Hedberg, J. Am. Chem. Soc., 1974, 96, 289.

2 R. Gleiter and R. Gygax, Top. Curr. Chem., 1976, 63, 1.

3 K. T. Potts, S. A. Nye and K. A. Smith, J. Org. Chem., 1992, 57, 3895; U. Chiacchio, A. Corsaro, A. Rescifina, M. G. Testa and G. Purrello, Heterocycles, 1993, 36, 223; H. G. Marei and M. M. Mishrikey, Phosphorus, Sulfur and Silicon, 1992, 73, 229.

4 K. Maeda, Bull. Chem. Soc. Japan, 1961, 34, 785; R. Cimiraglia and H.-J. Hofmann, J. Am. Chem. Soc., 1991, 113, 6449; A. S. Brown and V. H. Smith, J. Chem. Phys., 1993, 99, 1837.

5 H. Behringer, M. Ruff and R. Wiedenmann, Chem. Ber., 1964, 97, 1732; A. Hordvik, E. Sletten and J. Sletten, Acta Chem. Scand., 1966, 20, 2001; R. J. S. Beer, D. Cartwright and D. Harris, Tetrahedron Lett., 1967, 953.

6 R. J. S. Beer, D. Cartwright, D. Harris and R. J. Gait, J. Chem. Soc. C, 1971, 963; G. Duguay, D. H. Reid, K. O. Wade and R. G. Webster, J. Chem. Soc. C, 1971, 2829.

7 Y. Wang, M. J. Chen and C. H. Wu, Acta Crystallogr., Sect. B: Struct. Sci., 1988, 44, 179; K. J. Lin and Y. Wang, J. Phys. Chem., 1993, 97, 3176.

8 K. H. Yih, Y. C. Lin, M. C. Cheng and Y. Wang, J. Chem. Soc., Chem. Commun., 1993, 1380.

9 L. Pauling, The Nature of the Chemical Bond, 3rd ed., Cornell University Press, New York, 1960.

10 K. H. Yih, Y. C. Lin, M. C. Cheng and Y. Wang, Organometallics, 1994, 13, 1561. 